PROCEEDINGS OF THE

AMERICAN MATHEMATICAL SOCIETY

Volume 129, Number 8, Pages 2331-2342

S 0002-9939(01)05802-6

Article electronically published on January 23, 2001

\title{
SUR L'INÉGALITÉ DE POINCARÉ, À SUPPORT COMPACT, POUR UN OU PLUSIEURS CHAMPS DE VECTEURS
}

\author{
MAKHLOUF DERRIDJ
}

(Communicated by David S. Tartakoff)

\begin{abstract}
Nous démontrons, dans cette note, une inégalité de type Poincaré pour un ou plusieurs champs de vecteurs $\left\{X_{j}\right\}_{j=1, \ldots, r}, r \geq 1$, et des fonctions régulières à support contenu dans un voisinage d'une hypersurface $S$, sous une hypothèse naturelle de contact entre $S$ et la famille $\left\{X_{j}\right\}$. La constante intervenant dans cette inégalité est précisément reliée à l'épaisseur du voisinage autour de $S$ et à l'ordre du contact entre $S$ et $\left\{X_{j}\right\}$.
\end{abstract}

\section{INTRODUCTION}

Notre but, ici, est d'abord de donner une démonstration directe d'une inégalité de type Poincaré, précise, dans le cadre d'un champ de vecteur réel, de classe $C^{\infty}$ (où $C^{k}, k$ assez grand adapté à l'hypothèse faite sur ce champ: voir plus loin), dans un ouvert de $\mathbb{R}^{n}$.

Nous commençons par étudier le cas d'une bande $B_{d \xi}=\left\{\left\|x^{\prime}\right\|<d,\left|x_{n}\right|<\right.$ $\varepsilon\}$. Nous montrons, que sous une hypothèse naturelle reliant le champ $X$ et l'hypersurface $S=\left\{\left\|x^{\prime}\right\|<d,\left|x_{n}\right|=0\right\}$ (ici $x^{\prime}=\left(x_{1}, \ldots, x_{n-1}\right)$ ), nous pouvons majorer $\|u\|_{L^{p}\left(B_{d / 2, \varepsilon}\right)}$ par $C_{\varepsilon}\|X u\|_{L^{p}\left(B_{d, \varepsilon}\right)} ; 1 \leq p \leq \infty, C_{\varepsilon}$ est en $\varepsilon^{\frac{1}{k}}$, si $\varepsilon<\varepsilon_{0}$ petit, et $\operatorname{supp}(u) \subset\left\{\left|x_{n}\right|<\varepsilon\right\}$, et $k$ lié à l'hypothèse faite, $\left(\mathrm{H}_{S, k}\right)$.

Nous pouvons déduire de ce résultat local, un résultat global, de même nature, dans le cadre d'une couronne $S_{\varepsilon}=\{x ; d(x, S)<\varepsilon\}$, où $S$ est une hypersurface compacte, de classe $C^{\infty}$ (ou $C^{k}, k$ assez grand) dans $\mathbb{R}^{n}$ et $X$ défini au voisinage de $S$.

Nous démontrons le même genre de résultats pour une famille de champs de vecteurs réels, de classe $C^{\infty}$ (ou $C^{k}, k$ assez grand), sous l'hypothèse qu'un crochet de longueur $k$, formé à partir des champs $X_{1}, \ldots, X_{r}$, soit transversal à $S$, au voisinage du point considéré dans $S$ (dans le cas local) ou qu'en tout point de $S$, on ait l'hypothèse ci-dessus (dans le cadre global d'une hypersurface compacte $S$ ).

Ce travail se justifie surtout par la précision des constantes qui interviennent et la méthode utilisée (à partir, en particulier, de formules établies par L. Hörmander dans [3], grâce à la formule de Campbell-Hausdorff). Aussi bien dans le cas d'un champ de vecteurs $X$ que dans celui d'une famille $X_{1}, \ldots, X_{r}$, nous étudions le flot (ou "un flot composé" dans le 2ème cas) du champ $X$ (ou des champs $X_{j}$ ) et montrons qu'il sort de la bande (ou couronne considérée) assez vite, en fonction de l'épaisseur. D'autre part, la contrainte faite sur $\varepsilon<\varepsilon_{0}$ (dans le cas de la bande, ou

Received by the editors December 2, 1999.

2000 Mathematics Subject Classification. Primary 37C10, 35H10, 35B45. 
d'une couronne, dans le cas global), ne dépend que des constantes intervenant dans l'expression des hypothèses faites entre $X$ et $S$ ou entre la famille $\left\{X_{j}, j=1, \ldots, r\right\}$ et $S$. Une hypothèse du même genre faite sur tout un voisinage donné de $S$ conduira à des résultats du même type avec moins de contrainte sur $\varepsilon$.

Signalons que la majoration étudiée, sans précision sur $C_{\varepsilon}$ est établie dans [1]. Signalons aussi que les inégalités dites de Poincaré, plus générales sont relatives à la majoration de la norme $L^{q}$ de $u-u_{B}$ (où $u_{B}$ est la moyenne de $u$ sur la boule considérée $B)$ par la somme des normes $L^{p}$ de $X_{j}\left(u-u_{B}\right)$, dans la même boule, établies dans le cadre des champs vérifiant l'hypothèse de L. Hörmander ([2], [4, [5]).

Signalons enfin que nos champs ne satisfont pas nécessairement l'hypothèse de L. Hörmander, mais seulement l'hypothèse $\left(\mathrm{H}_{\mathrm{k}, \mathrm{S}}\right)$ (voir plus loin), dans le cas d'une famille de champs.

Il serait intéressant de voir si cette étude sur les flots (du ou des champs de vecteurs considérés) permet aussi d'étudier des inégalités de Poincaré, plus générales.

\section{DÉfinitions - NOtATions - CAS D'Un Champ DE VECTEURS}

a). Nous considérons un morceau de bande dans $\mathbb{R}^{n}$, dont la variable usuelle est notée $x=\left(x_{1}, \ldots, x_{n-1}, x_{n}\right)=\left(x^{\prime}, x\right)$, définie par

$$
B_{d, c}=\left\{x \in \mathbb{R}^{n},\left|x_{n}\right|<c,\left\|x^{\prime}\right\|<d\right\} .
$$

Dans la suite, $c$ sera très petit et sera donc souvent noté $\varepsilon$ et $d$ sera petit mais fixé, en fonction du champ réel $X$ (ou des champs réels $X_{j}$, suivant le cas) donné dans une bande fixée $B_{\alpha, \beta}$. On notera $S$ l'hypersurface [Sur l'inégalité de Poincaré...]

$$
S=\left\{x_{n}=0\left\|x^{\prime}\right\|<\alpha\right\} .
$$

On considère un champ de vecteur $X$, réel, de classe $C^{\infty}$, qu'on supposera non nul sur $B_{\alpha, \beta}$ (si $X(0) \neq 0$, on est dans ce cas, en prenant $(\alpha, \beta)$ assez petits). Le cas intéressant pour nous est bien entendu le cas où $X$ n'est pas transverse à $S$, en 0 . On peut supposer alors (en divisant par le coefficient de $\partial_{x_{1}}$, supposé non nul en 0 ) que l'on a (en prenant peut-être $\alpha$ et $\beta$ encore plus petits)

$$
X=\partial_{x_{1}}+\sum_{j=2}^{n} a_{j} \partial_{x_{j}} \text { dans } B_{\alpha, \beta} .
$$

Nous supposons maintenant l'hypothèse suivante satisfaite:

$$
\left(\mathrm{H}_{k, S}\right): \text { Il existe } k \in \mathbb{N}, \quad(\text { minimal }), \text { t.q } X^{k}\left(x_{n}\right) \neq 0 .
$$

Remarquons que l'hypothèse $\left(\mathrm{H}_{k, S}\right)$ est de nature géométrique portant sur "l'ordre de tangence de $X$ avec $S$ ".

Considérons maintenant dans $B_{\alpha, \beta}$ le flot associé à $X$, noté $\varphi=\left(\varphi_{1}, \ldots, \varphi_{n}\right)$ donné pour $t<t_{0}$ par

$$
\left\{\begin{array}{l}
\varphi_{t}^{\prime}(t, x)=X(\varphi(t, x)), \quad 0 \leq t<t_{0}, \\
\varphi(0, x)=x
\end{array}\right.
$$

De façon plus concrète ici (2.4) se réécrit, d'après (2.2):

$$
\left\{\begin{array}{l}
\varphi_{1}^{\prime}(t, x)=1, \quad 0 \leq t<t_{0}, \\
\varphi_{j}^{\prime}(t, x)=a_{j}(\varphi(t x)), \quad j \geq 2, \\
\varphi(0, x)=x
\end{array}\right.
$$


Notre but sera de montrer que, sous l'hypothèse $\left(\mathrm{H}_{k, S}\right)$, si $\varepsilon$ assez petit, alors pour tout $x \in B_{\frac{\alpha}{2}, \varepsilon}$, la courbe intégrale de $X$ issue de $x$ sort de $B_{\frac{\alpha}{2}, \varepsilon}$, par $\left|x_{n}\right|=\varepsilon$ au bout d'un temps inférieur à $C_{0} \varepsilon^{\frac{1}{k}}$, où $C_{0}$ est une constante fixe dépendant de $X$ $\operatorname{sur} B_{\alpha, \beta}$.

Pour cela, nous allons d'abord montrer, que $X$ a une écriture spéciale, si l'on fait un changement de variables en $\left(x_{1}, \ldots, x_{n-1}\right)$ seulement (laissant ainsi $x_{n}$ invariant).

Proposition 1. On peut trouver un changement de variables $\left(x_{1}, \ldots, x_{n}\right) \rightarrow$ $\left(u_{1}\left(x^{\prime}\right), \ldots, u_{n-1}\left(x^{\prime}\right), x_{n}\right)$, au voisinage $V$ de 0 , tel que l'on ait, avec $u=$ $\left(u_{1}, \ldots, u_{n-1}\right)$

$$
\left\{\begin{array}{l}
X=\partial_{u_{1}}+b_{n}(u) \partial_{x_{n}}+x_{n} Y \\
\text { où } Y \text { est un champ de vecteurs dans } V .
\end{array}\right.
$$

Démonstration. Soit donc $X$ donné par (2.2). Écrivons d'abord $a_{j}\left(x^{\prime}, x_{n}\right)=a_{j}\left(x^{\prime}, 0\right)$ $+x_{n} \tilde{a}_{j}(x)$. Ainsi on obtient

$$
\left\{\begin{aligned}
X & =\partial_{x_{1}}+\sum_{j=2}^{n} a_{j}\left(x^{\prime}, 0\right) \partial_{x_{j}}+\sum_{j=2}^{n} x_{n} \tilde{a}_{j}(x) \partial_{x_{j}} \\
& =\partial_{x_{1}}+\sum_{j=2}^{n-1} a_{j}\left(x^{\prime}, 0\right) \partial_{x_{j}}+a_{n}\left(x^{\prime}, 0\right) \partial_{x_{n}}+x_{n} \sum_{j=2}^{n} \tilde{a}_{j}(x) \partial_{x_{j}} .
\end{aligned}\right.
$$

Nous savons qu'il existe un changement de variables: $\left(x_{1}, \ldots, x_{n-1}\right) \rightarrow\left(u_{1}, \ldots, u_{n-1}\right)$ (rectification d'un champ de vecteurs) dans $\mathbb{R}^{n-1}$, près de $0 \in \mathbb{R}^{n-1}$, tel que

$$
\partial_{x_{1}}+\sum_{j=2}^{n-1} a_{j}\left(x^{\prime}, 0\right) \partial_{x_{j}}=\partial_{u_{1}} .
$$

Considérons alors le changement associé $(x) \rightarrow\left(u, x_{n}\right)$, près de $0 \in \mathbb{R}^{n}$. On obtient alors avec les variables $\left(u, x_{n}\right)$ :

$$
\left\{\begin{array}{l}
X=\partial_{u_{1}}+b_{n}(u) \partial_{x_{n}}+x_{n} Y \\
\operatorname{avec} Y=\sum_{j=1}^{n-1} \theta_{j}\left(u, x_{n}\right) \partial_{u_{j}}+\theta\left(u, x_{n}\right) \partial_{x_{n}} \text { dans } V
\end{array}\right.
$$

Donc, dorénavant, on suppose qu'avec $\left(x_{1}, \ldots, x_{n}\right)$ on a

$$
\left\{\begin{array}{l}
X=\partial_{x_{1}}+a\left(x^{\prime}\right) \partial_{x_{n}}+x_{n} Y \\
Y \text { champ de vecteurs dans } B_{\alpha, \beta} .
\end{array}\right.
$$

Proposition 2. Soit $X$ donné par (2.9) dans $B_{\alpha, \beta}$. Alors on a, sous l'hypothèse $\mathrm{H}_{k, S}$ :

$$
\frac{\partial^{k-1} a}{\partial x_{1}^{k-1}}(0) \neq 0 \quad \text { et } \quad \frac{\partial^{\ell} a}{\partial x_{1}^{\ell}}(0) \neq 0, \quad 0 \leq \ell \leq k-2 .
$$

Démonstration. Exprimons $X\left(x_{n}\right)$ et $X^{2}\left(x_{n}\right)$

$$
\begin{aligned}
X\left(x_{n}\right) & =a\left(x^{\prime}\right)+x_{n} Y\left(x_{n}\right), \\
X^{2}\left(x_{n}\right) & =\frac{\partial a}{\partial x_{1}}\left(x^{\prime}\right)+a Y\left(x_{n}\right)+x_{n} f_{2}(x) .
\end{aligned}
$$


Supposons, par récurrence, que:

$$
X^{p}\left(x_{n}\right)=\frac{\partial^{p-1} a}{\partial x_{1}^{p-1}}\left(x^{\prime}\right)+\sum_{\ell=0}^{p-2} \theta_{\ell} \frac{\partial^{\ell} a}{\partial x_{1}^{\ell}}\left(x^{\prime}\right)+x_{n} f_{p} .
$$

Appliquons $X$ à (2.11). On obtient:

$$
\begin{aligned}
X^{p+1}\left(x_{n}\right)= & \frac{\partial^{p} a}{\partial x_{1}^{p}}\left(x^{\prime}\right)+\sum_{\ell=0}^{p-2} \theta_{\ell} \frac{\partial^{\ell+1} a}{\partial x_{1}^{\ell+1}}\left(x^{\prime}\right)+a\left(x^{\prime}\right) \sum_{\ell=0}^{p-2} \partial_{x_{n}}\left(\theta_{\ell}\right) \\
& \cdot \frac{\partial^{\ell} a}{\partial x_{1}^{\ell}}\left(x^{\prime}\right)+a\left(x^{\prime}\right) f_{p}+x_{n} Y\left(X^{p}\left(x_{n}\right)\right)+\sum_{\ell=0}^{p-2} \frac{\partial \theta_{\ell}}{\partial x_{1}} \cdot \frac{\partial^{\ell} a}{\partial x_{1}^{\ell}}\left(x^{\prime}\right) \\
= & \frac{\partial^{p} a}{\partial x_{1}^{p}}\left(x^{\prime}\right)+\sum_{\ell=0}^{p-1} \theta_{\ell}^{\prime}(x) \frac{\partial^{\ell} a}{\partial x_{1}^{\ell}}\left(x^{\prime}\right)+x_{n} f_{p+1} .
\end{aligned}
$$

Ainsi la formule (2.11) est vérifiée. En utilisant l'hypothèse $\left(\mathrm{H}_{k, S}\right)$ on tire bien (2.10).

Proposition 3. Soit $X$ donné par (2.9) dans $B_{\alpha, \beta}$, et soit $\varphi$ le flot de $X$ dans $B_{\alpha, \beta}$, alors:

$$
\left\{\begin{array}{l}
\varphi_{1}(t, x)=x_{1}+\int_{0}^{t}\left[1+\varphi_{n}(s, x) \cdot b_{1}(\varphi(s, x))\right] d s, \quad 0 \leq t \leq t_{0} \\
\left.\varphi_{j}(t, x)=x_{j}+\int_{0}^{t} \varphi_{n}(s, x) \cdot b_{j}(\varphi(s, x))\right] d s, \quad 2 \leq j \leq n-1 \\
\varphi_{n}(t, x)=x_{n}+\int_{0}^{t}\left[a\left(\varphi_{1}(s, x), \ldots, \varphi_{n-1}(s, x)\right)+\varphi_{n}(s, x) \cdot b_{n}(\varphi(s, x))\right] d s \\
\text { où } Y=\sum_{j-1}^{n} b_{j}(x) \partial_{x_{j}}
\end{array}\right.
$$

Démonstration. Cela provient directement de (2.5), en tenant compte de l'expression de $X$ donnée dans (2.9).

Proposition 4. Soit $X$ donné par (2.9) dans $B_{\alpha, \beta}$, satisfaisant l'hypothèse $\left(\mathrm{H}_{k, S}\right)$. Alors, on a dans $B_{\alpha, \beta}, 0 \leq t \leq t_{0}$,

$$
\left\{\begin{array}{l}
\varphi_{\ell}(t, x)=x_{\ell}+\sum_{j=1}^{k} c_{\ell, j}(x) t^{j}+0\left(t^{k+1}\right), \quad 1 \leq \ell \leq n, \\
c_{11}(x) \neq 0 \quad \text { en fait } c_{11}(0)=1, \\
c_{n, k}(x) \neq 0, \quad c_{n, j}(0)=0, \quad j \leq k-1 .
\end{array}\right.
$$

Démonstration. Nous allons, bien entendu, exploiter l'écriture du champ $X$ (écriture $(2.9)$ ).

Nous pouvons toujours écrire $\varphi_{\ell}(t, x)$ sous la forme donnée dans (2.13) si l'on n'exige pas $c_{11} \neq 0$ et $c_{n, k} \neq 0$.

Montrons que l'hypothèse $\left(\mathrm{H}_{k, S}\right)$ entraîne que $c_{11}(x) \neq 0$ et $c_{n, k}(x) \neq 0$.

Il nous suffit de le faire en prenant $x=0$, (par continuité des $c_{\ell_{j}}$, en $x$ ): alors (2.13) sera montré, en prenant si nécessaire $\alpha$ et $\beta$ plus petits. Notons alors:

$\tilde{\varphi}_{j}(t)=\varphi_{j}(t, 0)$. Nous obtenons ainsi

$$
\left\{\begin{array}{l}
\tilde{\varphi}_{1}(t)=\int_{0}^{t}\left[1+\tilde{\varphi}_{n}(s) \cdot b_{1}(\tilde{\varphi}(s))\right] d s, \quad 0 \leq t<t_{0}, \\
\tilde{\varphi}_{j}(t)=\int_{0}^{t} \tilde{\varphi}_{n}(s) \cdot b_{j}(\tilde{\varphi}(s)) d s, \\
\left.\tilde{\varphi}_{n}(t)=\int_{0}^{t}\left[a \tilde{\varphi}_{1}(s), \ldots, \tilde{\varphi}_{n}(s)\right)+\tilde{\varphi}_{n}(s) b_{n}(\tilde{\varphi}(s))\right] d s .
\end{array}\right.
$$

Comme $\tilde{\varphi}_{n}(0)=0$, on a $\tilde{\varphi}_{1}(t)=t+0\left(t^{2}\right)$. On a donc $c_{11}(0)=1$, ce qui entraîne la première condition. Considérons maintenant l'expression de $\tilde{\varphi}_{n}(t)$ donnée dans 
(2.14). Dans l'intégrale, remplaçons $a(\tilde{\varphi}(s))$ par:

$$
\begin{gathered}
a(\tilde{\varphi}(s))=\frac{\partial^{k-1} a}{\partial x_{1}^{k-1}}(0)\left(\tilde{\varphi}_{1}(s)\right)^{k-1}+\sum_{j=2}^{n-1} \tilde{\varphi}_{j}(s) \cdot \theta_{j}(\tilde{\varphi}(s))+0\left(s^{k}\right), \\
\left\{\begin{array}{c}
\tilde{\varphi}_{n}(t)=\int_{0}^{t}\left[\sigma\left(s+\sum_{j=2}^{k} c_{1, j} s^{j}+0\left(s^{k+1}\right)\right)^{k-1}\right. \\
\left.+\sum_{j=2}^{n-1} \tilde{\varphi}_{j}(s) \cdot \theta_{j}(\tilde{\varphi}(s))+\tilde{\varphi}_{n}(s) b_{n}(\tilde{\varphi}(s))+0\left(s^{k}\right)\right] d s \\
\text { avec } \sigma=\mid \frac{\partial^{k-1} a}{\partial x_{1}^{k-1}(0) \mid} \neq 0 .
\end{array}\right.
\end{gathered}
$$

On aboutit alors, compte-tenu de (2.14) et (2.15) à

$$
\tilde{\varphi}_{n}(t)=\sigma t^{k}+0\left(t^{k+1}\right)+\int_{0}^{t} 0\left(\left|\tilde{\varphi}_{n}(s)\right|\right) d s .
$$

L'estimation (2.16) donne bien $\tilde{\varphi}_{n}(t)=\sigma t^{k}+0\left(t^{k+1}\right)$. Ainsi la proposition 4 est démontrée.

Proposition 5. Il existe $\varepsilon_{0}>0$, et $C_{0}>0$ tels que, pour $\varepsilon \leq \varepsilon_{0}$, la trajectoire de $X$, issue de tout point $x \in B_{\frac{\alpha}{2}, \varepsilon}$, atteint la frontière $\left\{\left|x_{n}\right|=\varepsilon\right\}$ en un temps $t_{x}$ inférieur à $C_{0} \varepsilon^{1 / k}$. De plus la trajectoire $\varphi(t, x), 0 \leq t \leq t_{x}$, est entièrement contenue dans $B_{d+C_{1} \varepsilon^{1 / k}, \varepsilon}\left(d=\frac{\alpha}{2}\right)$ avec $C_{1}$ constante convenable.

Démonstration. D'après la proposition 4 , on a, avec $0\left(t^{k+1}\right)$ uniformément

$$
\varphi_{n}(t, x)=x_{n}+\sum_{j=1}^{k} c_{n j}(x) t^{j}+0\left(t^{k+1}\right) \quad, \quad c_{n k} \neq 0,
$$

$\operatorname{sur} B_{\alpha, \beta}$ et $c_{n j}(0)=0, \quad j \leq k-1$.

Montrons d'abord que l'on a la propriété suivante:

$$
\left\{\begin{array}{l}
\text { Il existe une constante } C_{1}>0, C_{1}=C_{1}\left(X, B_{\alpha, \beta}\right), \varepsilon_{0}>0 \\
\text { tel que } \forall \varepsilon<\varepsilon_{0}, \forall x \in B_{\frac{\alpha}{2}, \varepsilon}\left|\sum_{j=1}^{k} c_{n j}(x) t_{x}^{j}+x_{n}\right|>2 \varepsilon, \\
\text { pour un } t_{x} \in\left[0, C_{1} \varepsilon^{1 / k}\right] .
\end{array}\right.
$$

On n'oubliera pas à la fin d'exiger $C_{1} \varepsilon^{1 / k}<t_{0}$ (inégalités (2.23)). Soit $x \in B_{\frac{\alpha}{2}, \varepsilon}$. Alors $\left|x_{n}\right|<\varepsilon$. On voit alors qu'il suffit de trouver $C_{1}>0, \varepsilon_{0}>0$ (indépendants de $\left.x \in B_{\frac{\alpha}{2}, \varepsilon}\right)$ tel que si $\varepsilon<\varepsilon_{0}$ alors

$$
\sup _{t \in\left[0, C_{1} \varepsilon^{1 / k}\right]}\left|\sum_{j=1}^{k} c_{n j}(x) t^{j}\right|>3 \varepsilon .
$$

Nous savons qu'il existe une constante $C=C(k)>0$ tel que

$$
\sup _{j=1, \ldots, k}\left|\theta_{j}\right| \leq C(k) \sup _{t \in[0,1]}|P(t)| \quad, \quad P=\sum_{j=1}^{k} \theta_{j} t^{j} .
$$


Nous tirons de là que, pour $C>0$ :

$$
\begin{aligned}
\sup _{t \in\left[0, C \varepsilon^{1 / k}\right]}|P(t)| & =\sup _{s \in[0,1]}\left|\sum_{j=1}^{k} \theta_{j} C^{j} \varepsilon^{j / k} s^{j}\right| \\
& \geq \frac{1}{C(k)} \sup _{j=1, \ldots, k}\left|\theta_{j} C^{j} \varepsilon^{j / k}\right| .
\end{aligned}
$$

En revenant à notre polynôme, on obtient:

$$
\sup _{t \in\left[0, C \varepsilon^{1 / k}\right]}\left|\sum_{j=1}^{k} c_{n j}(x) t^{j}\right| \geq \frac{1}{C(k)} \sup _{j=1, \ldots, k}\left|c_{n j}(x) C^{j} \varepsilon^{j / k}\right| .
$$

Comme $B_{\alpha, \beta}$ a été choisi de sorte que $\left|c_{n k}(x)\right| \geq \frac{\sigma}{2}>0$ sur $B_{\alpha, \beta}$ (indépendamment de $\varepsilon$ bien entendu car $\left.c_{n, k}(0)=\sigma\right)$.

On aboutit ainsi à:

$$
\sup _{t \in\left[0, C \varepsilon^{1 / k}\right]}\left|\sum_{j=1}^{k} c_{n j}(x) t^{j}\right| \geq \frac{1}{C(k)} \frac{\sigma}{2} C^{k} \varepsilon .
$$

On choisit alors $C_{1}$ de sorte que

$$
C_{1}^{k}>\frac{6 C(k)}{\sigma}
$$

On tire alors aisément de (2.20) et (2.21), la propriété (2.17), lorsqu'on n'a pas $0\left(t^{k+1}\right)$; d'autre part $\left|0\left(t^{k+1}\right)\right| \leq C_{2} t^{k+1} C_{2}$ uniforme sur $B_{\alpha, \beta}$. Nous tirons alors de (2.17), la minoration suivante:

$$
\begin{cases}\sup _{t \in\left[0, C_{1} \varepsilon^{1 / k}\right]}\left|\varphi_{n}(t, x)\right| & >2 \varepsilon-C_{2} C_{1}^{k+1} \varepsilon^{\frac{k+1}{k}} \\ & >\varepsilon\left(2-C_{2} C_{1}^{k+1} \varepsilon^{1 / k}\right) .\end{cases}
$$

Choisissons alors $\varepsilon_{0}$ de sorte que

$$
C_{2} C_{1}^{k+1} \varepsilon_{0}^{1 / k}<1 \quad \text { et } \quad C_{1} \varepsilon_{0}^{1 / k}<t_{0} .
$$

Alors la proposition (2.17) découle de (2.22) et (2.23). Pour terminer la démonstration de la proposition 5, il nous suffit d'estimer $\left|\varphi_{j}(t, x)\right|, j \leq n-1$, pour $t \in$ $\left[0, C_{1} \varepsilon^{1 / k}\right]$.

Nous avons, d'après (2.14)

$$
\left\{\begin{array}{lll}
\varphi_{1}(t, x) & = & t+x_{1}+0\left(t^{2}\right), \quad 0\left(t^{2}\right) \text { uniforme en } x \in B_{\alpha, \beta} \\
\varphi_{j}(t, x) & = & x_{j}+0\left(\varphi_{n}(t, x)\right), \quad 2 \leq j \leq n-1
\end{array}\right.
$$

On en tire alors la propriété suivante, sous la condition (2.23):

$$
\sup _{t \in\left[0, C_{1} \varepsilon^{1 / k}\right]}\left|\varphi_{j}(t, x)\right| \leq \frac{\alpha}{2}+C_{3} \varepsilon^{1 / k} \leq \alpha ; \quad \text { si } \varepsilon_{0} \leq\left(\frac{\alpha}{2 C_{3}}\right)^{k} ; \quad x \in B_{\frac{\alpha}{2}, \varepsilon} .
$$

Quitte à changer la notation de $C_{1}$ et $C_{3}$ en $C_{0}$ et $C_{1}$, la proposition 5 est entièrement démontrée. Dorénavant on considèrera $\varepsilon_{0}$ avec les contraintes (2.23) et (2.25). 
Nous sommes prêts, maintenant, pour démontrer le

Théorème 1. Soient $\varepsilon_{0}>0$ et $C_{0}>0$ tels que les conclusions de la proposition 5 soient satisfaites. Alors on a, pour $\varepsilon<\varepsilon_{0}$

$$
\left\{\begin{array}{l}
\|u\|_{L^{p}\left(B_{\left.\frac{\alpha}{2}, \varepsilon\right)}\right.} \leq C_{1} \varepsilon^{1 / k}\|X u\|_{L^{p}\left(B_{\frac{\alpha}{2}+C_{0} \varepsilon, \varepsilon}\right)}, \quad 1 \leq p \leq \infty, \\
u \in C^{\infty}\left(B_{\alpha, \varepsilon}\right), \operatorname{supp} u \subset\left\{\left|x_{n}\right|<\varepsilon\right\} .
\end{array}\right.
$$

Démonstration. Plaçons-nous dans les conditions précédentes.

Soit $u \in C^{\infty}\left(B_{\alpha, \varepsilon}\right)$ avec supp $u \subset\left\{\left|x_{n}\right|<\varepsilon\right\}$. Prenons $1<p<\infty$. Nous allons intégrer d'abord le long des trajectoires de $X$

$$
u\left(\varphi\left(t_{x}, x\right)\right)-u(x)=\int_{0}^{t_{x}} X u(\varphi(t, x)) d t .
$$

Comme $u\left(\varphi\left(t_{x}, x\right)\right)=0$, on tire de (2.27) l'estimation

$$
|u(x)| \leq \int_{0}^{C_{0} \varepsilon^{1 / k}}|X u(\varphi(t, x))| d t, \quad \operatorname{car} t_{x} \leq C_{0} \varepsilon^{1 / k} .
$$

Par l'inégalité de Hölder, il découle:

$$
|u(x)|^{p} \leq\left(C_{0} \varepsilon^{1 / k}\right)^{\frac{1}{q}} \int_{0}^{C_{0} \varepsilon^{1 / k}}|X u(\varphi(t, x))|^{p} d t ; \quad \frac{1}{q}+\frac{1}{p}=1 .
$$

Et, ensuite, en intégrant en $x$ :

$$
\int_{x \in B_{\frac{\alpha}{2}, \varepsilon}}|u(x)|^{p} d x \leq\left(C_{0} \varepsilon^{1 / k}\right)^{\frac{p}{q}} \int_{0}^{C_{0} \varepsilon^{1 / k}}\left(\int_{x \in B_{\frac{\alpha}{2}, \varepsilon}}|X u(\varphi(t, x))|^{p} d x\right) d t .
$$

On a utilisé le théorème de Fubini, pour avoir 2.30). Maintenant, à $t$ petit $\left(t<t_{0}\right.$ petit), le Jacobien de l'application:

$$
x \longrightarrow \varphi(t, x)
$$

est voisin de $1, \operatorname{car} \varphi(0, x)=x$. Par suite on obtient

$$
\int_{x \in B_{\frac{\alpha}{2}, \varepsilon}}|u(x)|^{p} d x \leq C_{2}\left(C_{0} \varepsilon^{1 / k}\right)^{\frac{p}{q}} \int_{0}^{C_{0} \varepsilon^{1 / k}}\left(\int_{\varphi \in B_{\frac{\alpha}{2}+C_{0} \varepsilon^{1 / k, \varepsilon}}}|X u(\varphi)|^{p} d \varphi\right) d t .
$$

Dans (2.31), le fait que $\varphi \in B_{\frac{\alpha}{2}+C_{0} \varepsilon^{1 / k}, \varepsilon}$, provient de l'estimation sur les $\varphi_{j}$ (Proposition 5): Or on a, trivialement

$$
\int_{0}^{C_{0} \varepsilon^{1 / k}}\left(\int_{\varphi \in B_{\frac{\alpha}{2}+C_{0} \varepsilon^{1 / k}, \varepsilon}}|X u(\varphi)|^{p} d \varphi\right) d t \leq C_{0} \varepsilon^{1 / k} \int_{B_{\frac{\alpha}{2}+C_{0} \varepsilon^{1 / k}, \varepsilon}}|X(u)|^{p} .
$$

Prenant alors $C_{1}$ convenable, on obtient bien (2.26).

Les cas $p=1$ et $p=\infty$ se font, à partir de (2.28) sans utiliser Hölder, de la même manière.

Remarques. a) En regardant de plus près, on peut spécifier les constantes $C_{0}, C_{1}, C_{2}$ entrant dans les estimations en fonction du champ de vecteur $X$ et de l'hypersurface $\left\{x_{n}=0\right\}$.

b) On pourrait établir un théorème global sur la bande $B_{\varepsilon}=\left\{x \in \mathbb{R}^{n} ;\left|x_{n}\right|<\varepsilon\right\}$, avec une hypothèse de "transversalité uniforme" de $X$ par rapport à $S=\left\{x_{n}=0\right\}$.

c) On peut se contenter de "champ $X$ de classe $C^{k}$ ". 
b) Cas d'une couronne autour d'une hypersurface compacte $S$ dans $\mathbb{R}^{n}$. Soit $S$ le bord d'un domaine $\Omega$ régulier de classe $C^{\infty}$. On notera alors pour $\varepsilon>0$ petit par:

$$
S_{\varepsilon}=\left\{x \in \mathbb{R}^{n} ; d(x, S)<\varepsilon\right\} . \quad \text { De plus } S=\{x ; \rho(x)=0\} .
$$

On prendra un champ de vecteurs défini au voisinage de $S$, (qui est supposée compacte $\left(\Omega\right.$ borné)), réel de classe $C^{\infty}$. Supposons que pour tout point $x_{0} \in S$, on a:

$\left(\mathrm{H}_{k_{x_{0}}, S}\right)$ : Il existe $k_{x_{0}}$, minimal, tel que $X^{k_{x_{0}}}(\rho)\left(x_{0}\right) \neq 0$.

Définissons alors $k$ par

$$
\left(\mathrm{H}_{k, S}\right): k=\sup _{x_{0} \in S} k_{x_{0}} .
$$

Il faut remarquer que si $\left(\mathrm{H}_{\left.k_{x_{0}}, S\right)}\right.$ est satisfaite, alors on a $X^{k_{0}} \rho(x) \neq 0$, pour $x$ voisin de $x_{0}$ : ainsi $k$ est fini, par compacité de $S$. On a alors le:

Théorème 2. Supposons que $k$ défini par (2.34) soit fini. Il existe $\varepsilon_{0}>0$ et $C_{0}>0$ tels que $\forall \varepsilon<\varepsilon_{0}$ on a, pour $1 \leq p \leq \infty$,

$$
\|u\|_{L^{p}\left(S_{\varepsilon}\right)} \leq C_{0} \varepsilon^{1 / k}\|X u\|_{L^{p}\left(S_{\varepsilon}\right)} \quad \forall u \in \mathcal{D}\left(S_{\varepsilon}\right) .
$$

Démonstration. La démonstration consiste à se ramener localement, au cas d'une bande.

Soit $x_{0} \in S$ : par difféomorphisme local, on se ramène au cas d'une bande.

Un morceau de la couronne, près de $x_{0}$, se ramène à un ouvert contenant une bande $B_{\alpha, \beta}$. Si l'on prend dans ce morceau de couronne, $\varepsilon_{x_{0}}$ suffisamment petit, la fonction $\tilde{u}$, transportée de $u$ dans $B_{\alpha, \beta}$ sera à support dans $\left\{\left|x_{n}\right|<\tilde{\varepsilon}\right\}$, avec $\tilde{\varepsilon}$ suffisamment petit (relativement à $X$ et $S$ près de $x_{0}$ ) pour que (2.34) soit satisfait, (avec, de plus, $\tilde{\varepsilon} \sim \varepsilon$ ). Ainsi

$$
\|\tilde{u}\|_{L^{p}\left(B \frac{a}{2}, \tilde{\varepsilon}\right)} \leq \tilde{C}_{1}\left(x_{0}\right) \tilde{\varepsilon}^{1 / k}\|\tilde{X} \tilde{u}\|_{L^{p}\left(B_{\alpha, \tilde{\varepsilon})}\right)}, \quad \tilde{\varepsilon} \leq \tilde{\varepsilon}_{x_{0}}\left(\operatorname{car} k \geq k_{x_{0}}, \quad \forall x_{0}\right) .
$$

On voit alors qu'on peut faire une partition de $S_{\varepsilon}$ en un nombre fini d'ensembles $S_{\varepsilon, \ell}, \ell=1, \ldots, \ell_{0}$, tel que

$$
\|u\|_{L^{p}\left(S_{\varepsilon, \ell}\right)} \leq C_{1, \ell} \varepsilon^{1 / k}\|X u\|_{L^{p}\left(S_{\varepsilon}\right)}, \quad u \in \mathcal{D}\left(S_{\varepsilon}\right) .
$$

D'où l'on tire alors

$$
\|u\|_{L^{p}\left(S_{\varepsilon}\right.}=\sum_{\ell=1}^{\ell_{0}}\|u\|_{L^{p}\left(S_{\varepsilon, \ell}\right)} \leq \ell_{0}\left(\sup _{\ell=1 \ldots \ell_{0}} C_{1, \ell}\right) \varepsilon^{1 / k}\|X u\|_{L^{p}(\varepsilon)} .
$$

En prenant $C_{0}$ convenable on obtient ainsi (2.35).

Remarque. On peut se contenter, ici aussi, de prendre un champ $X$ et une hypersurface $S$ de classe $C^{k}$, où $k$ est donné par (2.34).

\section{Inégalité de Poincaré dans le cas de plusieurs champs de vecteurs}

Nous allons établir, dans le cas de plusieurs champs $X_{1}, \ldots, X_{r}$ réels, de classe $C^{\infty}$, des théorèmes de majoration, analogues à ceux de la partie II, sous une hypothèse faite sur les crochets de champs de vecteurs $X_{j}, j=1, \ldots, r$, analogue à celle de L. Hörmander, mais seulement dans la direction normale à l'hypersurface considérée $S$. 
3.1. Cas d'une bande. Nous allons donc d'abord considérer le cas d'une bande $B_{\alpha, \beta}$, au voisinage de laquelle sont donnés les champs $X_{j}, j=1, \ldots, r$, avec 0 comme centre de $B_{\alpha, \beta}$.

$$
\left(\mathrm{H}_{k, S}\right)\left\{\begin{array}{l}
\text { Il existe un crochet (de longueur minimale } k \text { ) formé avec } \\
\text { des champs de la famille }\left\{X_{1}, \ldots, X_{r}\right\}, \text { qui a une } \\
\text { composante non nulle sur } \partial_{x_{n}}, \text { en } 0 .
\end{array}\right.
$$

Nous considérons alors, comme dans 2] les flots associés $\varphi_{X_{j}}, j=1, \ldots, r$, que nous supposons définis dans $\left[0, t_{0}\right] \times B_{\alpha, \beta} \subset \mathbb{R} \times \mathbb{R}^{n}$ (quitte à prendre $\alpha, \beta$ plus petits). De plus, remarquons que l'on peut aussi supposer que $\left(\mathrm{H}_{k, S}\right)$ est satisfaite dans un voisinage de la bande $\overline{B_{\alpha, \beta}}$.

Nous allons maintenant définir les "flots composés"

$$
\varphi_{I}(t, x), \quad \text { où } I=\left(i_{1}, \ldots, i_{p}\right), \quad i_{j} \in\{1, \ldots, r\} .
$$

Nous allons montrer que pour $I$ convenable, le "flot composé" $\varphi_{I}(t, x)$ sort de la bande $B_{\alpha, \varepsilon}$, pour $\varepsilon$ assez petit et $x$ fixé dans $B_{\frac{\alpha}{2}, \varepsilon}$, par la frontière $\left\{\left|x_{n}\right|=\varepsilon\right\}$ au bout d'un temps borné par $C_{0} \varepsilon^{\frac{1}{k}}$.

Nous définissons $\varphi_{I}(t, x)$ pour $I=\left(i_{1}, \ldots, i_{p}\right)$ par:

$$
\varphi_{I}(t, x)=\varphi_{X_{i_{1}}}\left(t, \varphi_{X_{i_{2}}}\left(t, \ldots, \varphi_{X_{i_{p}}}(t, x)\right), \ldots\right) ; \quad 0 \leq t<t_{0} \text { petit. }
$$

Dans le cas d'un seul champ de vecteurs $X$, nous avons étudié $\varphi=\varphi_{X}$, dans la bande $B_{\alpha, \varepsilon}$. Nous allons faire la même chose avec $\varphi_{I}$. Pour cela, nous allons utiliser l'étude faite par L. Hörmander pour les champs $X_{I}$, à partir de la formule de Campbell-Hausdorff, dans son fameux papier [3], sur l'hypoellipticité des sommes de carrés de champs de vecteurs.

Partant de la formule de Campbell-Hausdorff, L. Hörmander ([3], pp. 161-162) a montré que si $x_{1}, \ldots, x_{\ell}$ sont des indéterminées et si $c=a d x_{1}, \ldots, a d x_{\ell-1}\left(x_{\ell}\right)$ alors on a

$$
e^{c}=\left(\prod_{j} e^{ \pm x_{i_{j}}}\right) e^{c_{1}}, \ldots, e^{c_{\gamma}} e^{\mu}
$$

où $\prod_{j} e^{ \pm x_{i_{j}}}$ est un produit de $\left(3 \times 2^{\ell-1}-2\right)$ facteurs du type $e^{ \pm x_{j}}$, chaque $c_{i}$ est un crochet formé à partir des $x_{j}$, de longueur supérieure à la longueur de $c$ et $\mu$ est une somme formelle de crochets de longueur supérieure à $N, N$ étant donné, à l'avance, grand.

Nous n'utiliserons pas toute la précision de cette formule.

Prenons maintenant, ici, les champs $t X_{i_{1}}, \ldots, t X_{i_{\ell}}$. Alors la formule (3.2) appliqué à $x_{i_{j}}=t X_{i_{j}}, j=1, \ldots, \ell$, nous permet d'écrire :

$$
\left\{\begin{array}{l}
\left(\prod_{j} e^{ \pm t X_{i j}}\right) \theta(t, x)=e^{t^{\ell} a d X_{i_{1}}, \ldots, a d X_{i_{\ell-1}}\left(X_{i_{\ell}}\right)} \\
\text { avec } \theta(t, x)=x+0\left(t^{\ell+1}\right), \quad \text { avec } 0 \text { uniforme en } x \in B_{\frac{\alpha}{2}, \frac{\beta}{2}} .
\end{array}\right.
$$

La dernière ligne provient de la formule 3.2 , avec $c_{j}$ et $\mu$ de longueur supérieure à $\ell$.

En notant $J=\left(i_{j}\right)_{j \in J}, i_{j}$ apparaissant dans $\prod_{j}$ en \pm , on a alors:

$$
\varphi_{J}(t, x)=\left(\varphi_{t^{\ell} a d X_{i_{1}}, \ldots, a d X_{i_{\ell-1}}\left(X_{i_{\ell}}\right)}(t, x)+0\left(t^{\ell+1}\right)\right)
$$

ceci découle aisément de (3.3). 
Supposons maintenant que c'est le champ $a d X_{i_{1}}, \ldots,\left(X_{i_{k}}\right)$ qui a une composante non nulle en 0 sur $\partial_{x_{n}}$, que l'on note $X_{I}$ : De (3.4) on tire alors, avec $J$ donné par (3.3)

$$
\left\{\begin{array}{l}
\varphi_{J}(t, x)=\varphi_{t^{k} X_{I}}(t, x)+0\left(t^{k+1}\right) \\
\text { avec } X_{I} \text { transverse à } S=\left\{x_{n}=0\right\} .
\end{array}\right.
$$

Il faut bien distinguer dans (3.5) entre $\varphi_{J}$, qui est un flot composé et $\varphi_{t^{k} X_{I}}$ qui est le flot du champ $t^{k} X_{I}$.

Ici on s'est ramené, du point de vue géométrique à un cas simple, à savoir le flot d'un champ transverse $t^{k} X_{I}$, modulo $0\left(t^{k+1}\right)$ qui est uniforme en $x \in B_{\frac{\alpha}{2}, \frac{\beta}{2}}$.

Ainsi pour $x$ fixé, la courbe $t \in\left[0, t_{0}\right] \rightarrow \varphi_{J}(t, x)$ sort de $B_{\alpha, \varepsilon}$, si $\varepsilon$ assez petit au bout d'un temps majoré par $C_{0} \varepsilon^{\frac{1}{k}}$ ( car $X_{I}$ est transverse et on considère $t^{k} X_{I}$ ), avec $C_{0}$ convenable, donné par la transversalité de $X_{I}$ (donc indépendante de $\varepsilon$ ). Nous notons de nouveau par $t_{x}$ l'instant où $\left|\left(\varphi_{J}\right)_{n}(t, x)\right|=\varepsilon$. De plus, d'après (3.5), pour $x \in B_{\frac{\alpha}{2}, \varepsilon}, \varepsilon<\varepsilon_{0}$, on a $\varphi_{J}(t, x) \in B_{\frac{\alpha}{2}+C_{0} \varepsilon, \varepsilon}$ si $0 \leq t \leq t_{x}$. De tout cela, nous tirons le

Théorème 3. Supposons que la famille $\left\{X_{1}, \ldots, X_{r}\right\}$ satisfait l'hypothèse $\left(\mathrm{H}_{k, S}\right)$ dans $B_{\alpha, \beta}$. Il existe $\varepsilon_{0}>0, C_{1}>0$ et $C_{0}>0$ tels que l'on ait, avec $1 \leq p \leq \infty$

$$
\left\{\begin{array}{l}
\|u\|_{L^{P}\left(B_{\left.\frac{\alpha}{2}, \varepsilon\right)}\right.} \leq C_{1} \varepsilon^{\frac{1}{k}} \sum_{j=1}^{r}\left\|X_{j} u\right\|_{L^{P}\left(B_{\frac{\alpha}{2}+C_{0} \varepsilon, \varepsilon}\right)}, \\
\text { si } u \in C^{\infty}\left(B_{\alpha, \varepsilon)}\right) \text { et } \operatorname{supp}(u) \subset\left\{\left|x_{n}\right|<\varepsilon\right\} .
\end{array}\right.
$$

Démonstration. Pour $x$ fixé dans $B_{\frac{\alpha}{2}, \varepsilon}$ on a alors

$$
\left\{\begin{array}{l}
\varphi_{J}(t, x)=\varphi_{Y_{1}}\left(t_{x}, \varphi_{Y_{2}}\left(t_{x}, \ldots, \varphi_{Y_{\nu-1}}\left(t_{x}, \varphi_{y_{\nu}}\left(t_{x}, x\right), \ldots\right)\right)\right) \\
\text { où les champs } Y_{\ell}, \quad \ell=1, \ldots, \nu \\
\text { sont les champs } \pm t X_{J} \text { figurant dans (3.3). }
\end{array}\right.
$$

On peut donc considérer le point $\varphi_{J}(t, x)$, comme l'extrémité d'une courbe régulière par morceaux, chaque morceau étant un morceau de courbe intégrale d'un des champs $Y_{\ell}$.

On peut supposer que chacun de ces morceaux de courbe intégrale est dans $B_{\alpha, \varepsilon}$ (sinon on s'arrête dès que l'on sort de $B_{\alpha, \varepsilon}$ par $\left\{\left|x_{n}\right|=\varepsilon\right\}$ et dans la somme cidessous on aura moins de termes, ce qui est mieux). Nous nous plaçons dans ce cas où tous les morceaux sont dans $B_{\alpha, \varepsilon}$. On peut alors écrire:

$$
\left\{\begin{array}{l}
u\left(\varphi_{J}\left(t_{x}, x\right)\right)-u(x) \\
=u\left(\varphi_{Y_{1}}\left(t_{x}, \varphi_{Y_{2}}\left(t_{x}, \ldots, \varphi_{Y_{\nu-1}}\left(t_{x}, \varphi_{Y_{\nu}}\left(t_{x}, x\right)\right) \ldots\right)\right)-u\left(\varphi_{Y_{1}}\left(0, \varphi_{Y_{2}}\left(t_{x}, \ldots\right)\right)\right)\right. \\
\quad+u\left(\varphi_{Y_{2}}\left(t_{x}, \ldots, \varphi_{Y_{\nu}}\left(t_{x}, x\right) \ldots\right)\right)-u\left(\varphi_{Y_{2}}(0, \ldots)\right) \\
\quad+\ldots \\
\quad \vdots \\
\quad+u\left(\varphi_{Y_{\nu}}\left(t_{x}, x\right)\right)-u\left(\varphi_{Y_{\nu}}(0, x)\right) .
\end{array}\right.
$$

Considérons maintenant $u$ telle que supp $u \subset\left\{\left|x_{n}\right|<\varepsilon\right\}$. Comme nous l'avons dit ci-dessus, on suppose que les morceaux de courbes intégrales sont dans $B_{\alpha, \varepsilon}$, sinon 
on ne prend que ceux qui sont dans $B_{\alpha, \varepsilon}$, ce qui ne peut qu'améliorer la majoration qui suit. Nous avons:

$$
\left\{\begin{aligned}
|u(x)| \leq & \int_{0}^{t_{x}}\left|Y_{1} u\left(s, \varphi_{Y_{2}}\left(t_{x}, \ldots, \varphi_{Y_{\nu-1}}\left(t_{x}, \varphi_{Y_{\nu}}\left(t_{x}, x\right)\right) \ldots\right)\right)\right| d s \\
& +\int_{0}^{t_{x}}\left|Y_{2} u\left(s, \varphi_{Y_{3}}\left(t_{x}, \ldots,\left(t_{x}, \varphi_{Y_{\nu}}\left(t_{x}, x\right)\right) \ldots\right)\right)\right| d s \\
& \vdots \\
& +\int_{0}^{t_{x}}\left|Y_{\nu} u(s, x)\right| d s
\end{aligned}\right.
$$

Nous tirons alors de (3.9), en prenant d'abord $1<p<\infty$,

$$
\begin{aligned}
|u(x)|^{p} \leq 2^{\nu}\left|t_{x}\right|^{\frac{p}{q}}\left(\int_{0}^{t_{x}}\left|Y_{1} u\left(s, \varphi_{Y_{2}}\left(t_{x}, \ldots, \varphi_{Y_{\nu}}\left(t_{x}, x\right)\right) \ldots\right)\right|^{p} d s\right. \\
\left.+\ldots+\int_{0}^{t_{x}}\left|Y_{\nu} u(s, x)\right|^{p} d s\right) \\
\leq 2^{\nu}\left(C_{0} \varepsilon^{\frac{1}{k}}\right)^{\frac{p}{q}}\left(\int_{0}^{C_{0} \varepsilon^{\frac{1}{k}}}\left|Y_{\nu} u(s, x)\right|^{p} d s\right. \\
\left.\quad+\ldots+\int_{0}^{C_{0} \varepsilon^{\frac{1}{k}}}\left|Y_{1} u\left(s, \ldots, \varphi_{Y_{\nu}}\left(t_{x}, x\right)\right)\right|^{p} d s\right) .
\end{aligned}
$$

Considérons une des intégrales: $\quad \int_{0}^{C_{0} \varepsilon^{\frac{1}{k}}}\left|Y_{j} u\left(s, \varphi_{Y_{j+1}}\left(t_{x}, \ldots, \varphi_{Y_{\nu}}\left(t_{x}, x\right)\right)\right)\right|^{p} d s$. Comme $t_{x}$ est voisin de $0\left(C_{0} \varepsilon^{\frac{1}{k}}\right.$ est voisin de 0$)$, nous voyons que, pour $t_{x} \in$ $\left[0, C_{0} \varepsilon^{\frac{1}{k}}\right], \ldots$, l'application:

$$
x \in B_{\frac{\alpha}{2}} \longrightarrow\left(\varphi_{Y_{j+1}}\left(t_{x}, \ldots, \varphi_{Y_{\nu}}\left(t_{x}, x\right)\right)\right) \in B_{\alpha, \varepsilon}
$$

a un Jacobien voisin de $1, \varepsilon<\varepsilon_{0}, \varepsilon_{0}$ assez petit.

Par suite, nous obtenons, avec une constante $C_{2}$ convenable:

$$
\int_{B_{\frac{\alpha}{2}, \varepsilon}}|u(x)|^{p} d x \leq C_{2}\left(\varepsilon^{\frac{1}{k}}\right)^{\frac{p}{q}} C_{0} \varepsilon^{\frac{1}{k}} \sum_{j=1}^{\nu} \int_{B_{\frac{\alpha}{2}+C_{0} \varepsilon, \varepsilon}}\left|Y_{j} u(y)\right|^{p} d y
$$

où $y$ joue le rôle de $\varphi_{Y_{\ell}}$ (voir l'inégalité (2.31)). Alors, avec $C_{1}$ convenable, (3.6) découle de (3.10).

Les cas $p=1$ et $p=\infty$, se font de la même manière, avec des majorations plus directes.

3.2. Cas d'une couronne autour d'une hypersurface compacte $S$. En utilisant, exactement, le même raisonnement que dans la partie 2, on peut montrer le théorème qui va suivre: Soit $S$ une hypersurface compacte dans $\mathbb{R}^{n}$ donnée par $\{\rho=0\}$. On considère dans un voisinage de $S$, disons $S_{d}=\left\{x \in \mathbb{R}^{n}, d(x, S)<d\right\}$, une famille de champs de vecteurs $X_{1}, \ldots, X_{r}$, réels de classe $C^{\infty}$. On suppose satisfaite l'hypothèse suivante pour $x_{0} \in S\left(\mathrm{H}_{k_{x_{0}}, S}\right)$ : il existe un crochet de longueur (minimale) $k_{x_{0}}$ qui est transverse à $S$ en $x_{0}$.

$\left(\mathrm{H}_{k, S}\right): k=\sup _{x_{0} \in S} k_{x_{0}}$ est fini, où $k_{x_{0}}$ donné ci-dessus. 
Théorème 4. Il existe $\varepsilon_{0}>0, C_{0}>0$, tels que $\forall \varepsilon<\varepsilon_{0}$ on ait, (sous l'hypothèse $\left(\mathrm{H}_{k, S}\right)$ ) et $1 \leq p \leq \infty$

$$
\|u\|_{L^{p}\left(S_{\varepsilon}\right)} \leq C_{0} \varepsilon^{\frac{1}{k}} \sum_{j=1}^{r}\left\|X_{j} u\right\|_{L^{p}\left(S_{\varepsilon}\right)} \quad \forall u \in \mathcal{D}\left(S_{\varepsilon}\right) .
$$

Comme nous l'avons dit (voir théorème 2) on raisonne localement, puis on utilise une partition de $S_{\varepsilon}$ en un nombre fini $S_{\varepsilon, \ell}$ de morceaux identifiés à des bandes, par difféomorphisme.

\section{REFERENCES}

[1] M. Derridj, J.-P. Dias. Le problème de Dirichlet pour une classe d'opérateurs non linéaires. J. Math. pures et appl. 51, (1972), p. 219-230. MR 54:8015

[2] B. Franchi, G. Lu, R.L. Wheeden. Representation formulas and weighted Poincaré inequalities for Hörmander vector fields. Ann. Inst. Fourier 45(2), (1995), p. 577-604. MR 96i:46037

[3] L. Hörmander. Hypoelliptic second order differential equations. Acta Math. 119 (1967), p. 147-171. MR 36:5526

[4] D. Jerison. The Poincaré inequalities for vector fields satisfying Hörmander's condition. Duke Math. J. 53, (1986), p. 503-523. MR 87i:35027

[5] G. Lu. Weighted Poincaré and Sobolev inequalities for vector fields satisfying Hörmander's condition. Revista Mat. Iberoamericana 8 (1992), p. 367-439. MR 94c:35061

Upresa Cnrs 6085, site Colbert, Université de Rouen, F76821 Mont Saint-Aignan, FRANCE

E-mail address: Makhlouf.Derridj@univ_rouen.fr 